\title{
MRI segmentation of medical images using FCM with initialized class centers via genetic algorithm
}

\begin{abstract}
Image segmentation is a critical stage in many computer vision and image process applications. Accurate segmentation of medical images is very essential in Medical applications but it is very difficult job due to noise and in homogeneity. Fuzzy C-Mean (FCM) is one of the most popular Medical image clustering methods. We noticed that for some images, FCM is sensitive to initialization of centre of clusters. This article introduced a new method based on the combination of genetic algorithm and FCM to solve this problem. The genetic algorithm is used to find initialized centre of the clusters. In this method, the centre is obtained by minimizing an object Function. This object Function specifies sum of distances between each data and their cluster centres. Then FCM is applied with to the case. The experimental result demonstrates the effectiveness of new method by able to initialize centre of the clusters.
\end{abstract}

